\title{
PERCEPCIÓN DEL CONOCIMIENTO DE LA DEMANDA DE PRODUCTOS Y PERCEPCIÓN HACIA LA INTERDEPENDENCIA ECONÓMICA EN EL CASERÍO QUERAL, CARAZ, ANCASH*
}

\author{
Mildred Paredes T. ${ }^{1}$, Héctor Hernández V. ${ }^{2}$, Luis Vicuña $P^{3}{ }^{3}$ Jesús De Miguel $C .{ }^{4}$ \\ (Recibido el 02/03/2007, aceptado el 11/04/2007)
}

In Memoriam al amigo Héctor Felipe Salverredy Marzal**

\begin{abstract}
RESUMEN
Objetivos: Identificar la percepción de los miembros del Caserío Queral hacia 1) la formación de relaciones interdependientes económicas y la percepción del conocimiento de la demanda de sus productos del Caserío Queral, 2) Conocer la relación entre la percepción del conocimiento de la demanda de productos con las percepciones hacia la interdependencia económica en la muestra de 51 miembros, un representante por familia mayor de 14 años del Caserío Queral. Diseño: Investigación aplicativa correlacional. Material y métodos: Datos obtenidos con dos escalas: Escala de Percepción a la Interdependencia Económica que mide tres factores independientes, y la Escala de Percepción al conocimiento a la Demanda que mide a partir de 6 factores con una confiabilidad de 0,93. Resultados: El conocimiento de la demanda es bajo, en tanto que la percepción de la interdependencia es favorable. Se encontró 5 correlaciones significativas, pero inversas entre componentes de dichos factores a excepción de la relación entre la Percepción del Conocimiento de la Oferta de Productos con Percepción hacia la Interdependencia económica por la Percepción de la Oferta $(0,29)$. Los restantes componentes no se correlacionan entre sí. Conclusión: No se encontró relación significativa $(-0,05)$ entre la Percepción del Conocimiento de la Demanda con Percepción hacia la Interdependencia Económica en el Caserío Queral en Caraz, Ancash.
\end{abstract}

Palabras clave: Percepción del conocimiento de la demanda, Percepción hacia la interdependencia económica, Conocimiento de la percepción de la oferta.

\footnotetext{
Docente Asociado de la Facultad de Psicología de la UNMSM. E-mail: mparedest@unmsm.edu.pe Docente Asociado de la Facultad de Psicología de la UNMSM. E-mail: hhvalz@gmail.com

Docente Principal de la Facultad de Psicología de la UNMSM. E-mail: luisvicunaperi@hotmail.com

4 Docente Asociado de la Facultad de Psicología de la UAM. E-mail: jesus.demiguel@uam.es

* El estudio se realizó con la colaboración de los alumnos de pregrado de la Facultad de Psicología: Roberto Aliaga y Miriam Laguna.

** Docente Auxiliar de la Facultad de Ingeniería Electrónica de la UNMSM.
} 


\begin{abstract}
Objectives: To identify the perception of Queral Small village's members towards the 1) formation of economic interdependent relations and the perception of knowledge about the demand of its products of the Queral Small village, 2) To know the relation between the perception of knowledge the demand of products with economic perceptions interdependence in the sample of 51 members, a representing one by greater family of 14 years of the Queral Small village. Design: Correlational applied investigation. Material and methods: Data collected with two scales: Scale of Perception to the Economic Interdependence that measures three independent factors, and the Scale of Perception to the knowledge to the demand, measures from 6 factors with a trustworthiness of 0,93. Results: The knowledge of the demand is low, whereas the perception of the interdependence is favorable. One was 5 significant but inverse correlations between components of these factors with the exception of the relation between the Perception of the Knowledge of the Supply of Products with Perception towards the economic Interdependence by the Perception of the Supply $(0,29)$. The component rest do not correlate to each other. Conclusion: Not significant relation (0.05) between the Perception of the Knowledge of the Demand with Perception towards the Economic Interdependence in the Queral Small village in Caraz, Ancash.
\end{abstract}

Keywords: Perception of the knowledge of the demand, Perception towards the economic interdependence, Knowledge of the perception of the supply,

\title{
INTRODUCCIÓN
}

El presente estudio en el Caserío de Queral, de la Comunidad Campesina Santa Cruz de Mayo, Caraz, se enmarca en el campo de estudio de la Psicología Social Organizacional y Psicología Económica, basándose en las investigaciones que se vienen haciendo desde el año 2003; antecedentes que se constituyen en aporte de la psicología al conocimiento de la realidad de la organización y gestión de la comunidad campesina localizada en la zona rural de los Andes peruanos del Callejón de Huaylas. Entender y proporcionar conocimiento científico sobre su comportamiento para contribuir a la formulación de planes dirigidos a un desarrollo sustentable, según las exigencias y necesidades de los propios actores, es una necesidad académica y profesional.

Teniendo como premisa que el Caserío de Queral se diferencia de los demás caseríos, al igual que una comunidad de otra, por su construcción social organizacional comunitaria sui generis; representación social que se va construyendo según la imagen (cognitiva) de sus miembros, quienes puntualizan prioritariamente a la Productividad, descrito por Paredes, M. \& De Miguel, J. Ma. (2004) [1]. En dicho microcontexto, la reprocidad norma pautando el comportamiento observable de compartir los productos básicos de la dieta diaria de manera equitativa, obtenidos de cosechas y crianza estacionaria, cuyo intercambio comercial está tradicionalmente establecido por la modalidad del trueque. Entre ellos comparten los menesteres de la vida cotidiana, social y cultural como parte de su identidad, otorgando un peculiar estilo de vida que se mantiene por su interacción.

La construcción social de la organización de la comunidad campesina poseedora de la calidad de dimensiones psicosociales que aparentemente varían, como es observable, en el comportamiento económico de transacción o comercialización con beneficio pecuniario, así, por ejemplo, la reciprocidad no es observable en el manejo de aquellos productos destinados a la comercialización ya que soslayan entre ellos la posibilidad de lograr volúmenes para su venta directa de manera conjunta o compartir las actividades dirigidas al lucro. 
Por otra parte, en Paredes, M. y col. (2005) [2] se registra que los miembros de la comunidad perciben adecuamente las condiciones de trabajo, a pesar de que pasan hambre durante el desarrollo de sus actividades del trabajo (faenas) $(0,18)$, perciben que sus recursos van de escasos a limitados $(0,2)$ para resolver lo inmediato en su economía de sobrevivencia; autodescriben un moderado manejo del medio ambiente $(0,3)$; son positivos para enfrentar el entorno $(0,39)$; y buscan mantener el diseño de sus construcciones con material ecológico (teja 0,4$)$

Son espacios reales que tiene la persona para aprender a socializarse, son los contextos microambientales de las organizaciones construidas, tales como instituciones, empresas, o la comunidad campesina andina, especialmente el caserío objeto de estudio, confirmado por los estudios psicológicos que señalan que su cultura propicia y genera aprendizajes de interacción interdependiente; ella sustenta comportamientos proactivos y productivos, indicándose, entre otras razones, porque posibilitan niveles de libertad cimentada en la interacción interdependiente, que por su naturaleza desarrolla la personalidad -caracterizada por factores de autoestima alta- y presencia significativa de la motivación de logro (nlogro) indicador del desarrollo económico, según McClelland (1970) [3], de un grupo o sociedad.

$\mathrm{Al}$ respecto, en nuestra realidad, no se ha identificado la presencia significativa de la $\mathrm{n}$ logro en el grupo de empresarios pesqueros exportadores, en el estudio de M. Paredes, M. (2001,127)[4]; disponiéndose más bien de datos significativos que la motivación de filiación (n-filiación) es la que sustenta la conducta. Así en el estudio en los alumnos de la UNMSM es significativa la relación entre n-filiación con la formación de empresas y la autogestión de su empleo, según alcances de Vicuña, L. y colaboradores. Por el contrario, en los micro contextos de socialización que propicien una interacción dependendiente se encontrará inmadurez, los actores optan por el sometimiento o acentuando la autopercepción de falta de dignidad en el intercabio de las relaciones humanas, entre otras, conclusión a que llega Argyris con respecto a las organizaciones con estructuras burócratas anquilosadas.

Por otro lado tenemos, en primer lugar, la dimensión de la identidad de las dimensiones psicosociales que describen a su construcción organizacional con respecto a la escala ordinal de Satisfación de la Comunidad Campesina Andina, está la dimensión actitudinal expresada en identificación que es condición para las conductas grupales de participación y cooperación, capital social, atribuidas y valoradas en los proyectos por el Banco Mundial.

Es necesario conocer, a partir de la percepción, la naturaleza de la interacción en el caserío para la eficiencia en las transacciones económicas sostenibles, posibilitando también un indicador fundamental para diseñar su estrategia y plantearse interrogantes más específicas para comprender mejor la incidencia de la motivación de filiación detectada en la población urbana.

Las preferencias individuales del mercado -usuarios del producto o servicio- sobre las cuales se basan las tendencias económicas no cambian frecuentemente y de súbito; si se producen, ellas también se sujetan al cambio del contexto social donde se desempeñan las personas en concordancia a su situación y oportunidades del entorno. En tal sentido Holahan, C. (1996) [5] señala que los contextos medioambientales precisan comportamientos y normas ajenos al individuo que moldean su conducta, y por otra parte Katona, G. (1965) [6] puntualiza que la conducta económica no está desligada de las variables psicosociales como 
la actitud, la misma que se forma de acuerdo a la conducta económica; adquirida la actitud, orienta o predispone el comportamiento de la persona, dándole una direccionalidad a su toma de decisiones económicas.

Al enfrentar actualmente la realidad del mercado o el entorno con orientación proactiva, la estrategia -entendida como la capacidad de respuesta de la organización hacia las exigencias del medio o contexto del mercado con características de lograr relaciones interdependientes(Paredes, M., 2001,111)[7] debe ser competitiva e indudablemente calificada por el tipo de interacción interdependiente de sus integrantes al construir la estructura del sistema social y en interacción con otros sistemas organizacionales, lo que es competencia de la organización social competitiva. La formulación de la estrategia competitiva se centra en estar orientada a aliarse, formar cadenas productivas y de comercialización según el comportamiento de la demanda y la real necesidad en la preservación del medio ambiente y la preocupación ecológica en el comportamiento. Desestimar este hecho es propiciar o ahondar la pobreza, (Paredes, M., 2002)[8].

La necesidad del conocimiento de la demanda de productos basada en la información de la percepción, actitud o motivación del comportamiento económico, es la partida para enriquecer o implementar o fortalecer una gestión prospectiva del sistema organizacional de la comunidad. Razón que nos encamina a encontrar la relación entre la percepción del conocimiento de la demanda de productos con las percepciones hacia la interdependencia económica en el Caserío Queral.

A fin de identificar la percepción de relación de interdependencia económica entre dos o más familias miembros del Caserío de Queral de la Comunidad Campesina de Santa Cruz, para proveer de criterios en la toma de decisión de los miembros mayores de 14 años por familia, en calidad de representantes, a propósito de proponer la instrumentación sustentada en la construcción organizacional de la comunidad que favorezca una gestión de calidad que reinvierta su economía de sobrevivencia en un progreso económico sustentable.

\section{Antecedentes}

A la Comunidad Campesina del Callejón de Huaylas se la viene estudiando a partir del año 2003 desde la competencia de la Psicología Social Organizacional. La investigación sobre Construcción social de la comunidad para el desarrollo del Callejón de Huaylas concluye que las dimensiones que la identifican en su representación social, a nivel de su imagen, son: Productividad, Identidad, Coordinación, Presión, entre las más significativas $(3,13$ 3,01 ), y en cuanto a la satisfacción puntean: Identidad, Productividad, Relaciones sociales, Motivación, Iniciativa, Coordinación, Valor de los recursos humanos, Grado de estructura impuesta $(3,17 ; 3,01)$. Continuando con el significativo aporte de este estudio, se ha identificado que el Caserío es la variable de agrupación con mayor poder para diferenciar la imagen que tienen los miembros de su comunidad, con pronóstico al 100\%. A estas características se las tipifica como Organización K (calidad psicológica) (Paredes, M. y De Miguel, J. Ma. 2004) [1].

La autopercepción a las condiciones en el trabajo en el caserío del estudio refiere como adecuadas: no sufrir accidentes y no enfermermarse $(0,3$ a 0,4$)$; aún cuando hay diferencia significativa a «pasar hambre» en el Caserío Queral $(1,8)$, lo describen como un entorno saludable, sin contaminantes $(0,4)$ aún cuando su economía no les posibilita subsanar gastos 
básicos por gestión de la comunidad $(0,2)$ señalan su manejo del medio ambiente $(0,4)$ por competencia de la comunidad (Paredes, M.; De Miguel, J. Mª; Vicuña, L. \& Hernández, H., 2005) [2].

Por comparación, la percepción de los empresarios exportadores pesqueros peruanos hacia modalidades de negocios compartidos alcanza una tendencia desfavorable, es decir, desestiman como posibilidad empresarial de realizar alianzas estratégicas: Joint-venture, tradings, franquicias preferentemente, conclusión, entre otras, de la investigación sobre la interdependencia empresarial (Paredes, M., 1997) [7].

El fenómeno de la globalización es un hecho en sí de carácter económico, como resultante del desarrollo tecnológico, el que trae consigo efectos socioculturales a cuidarse; de ahí que, ante tal situación, J. Ma. De Miguel (2004)[9] plantea que mediante el desarrollo local los pueblos y las personas pueden adaptarse con menos trauma y mayor posibilidad de éxito a esta nueva forma de la economía y de relaciones sociales a escala mundial. Fundamentando que el modelo de desarrollo local es una estrategia no sólo oportuna; por tratarse de un proceso continuo de mejora y cambio estructural que propicia el crecimiento económico, el incremento en la calidad de vida, la equidad, la participación social y la sostenibilidad; sino que también es significativa como alternativa a las secuelas sociales de la globalización, imposición de estructuras y, entre otras, el incremento de la desigualdad social, la destrucción de los recursos ambientales de los territorios, la colocación de barreras para acceder a los recursos que valorizan a las personas -conocimiento tecnológico y científico- o propiciar la cosificación de sociedad e individuos como elementos de mercado. Teniéndose:

El modelo integra tres elementos: 1) Objetivos del desarrollo local: desarrollo, un instrumento para la promoción de los derechos de las personas y los pueblos 2) las dimensiones psicosociales: lo local, la respuesta de los pueblos al desafío global y, dimensiones psicosociales, identidad, diversidad y conocimiento, 3) las líneas estratégicas: acciones de desarrollo, convertir el deseo en realidad. (De Miguel, J. Ma., 2004, 460) [9]

Uno de los aspectos clave dentro de las organizaciones es la generación y preservación del conocimiento, entre ellos se tiene el conocimiento de la demanda como necesidad en la gerencia prospectiva. Siempre que se entienda como lo señala Juanola i Hospital, Eduard (2004, 27-28) [10] «que nuestras acciones, percepción y decisiones económicas no se fundamentan tanto en la existencia de una conciencia racional, como en diferentes disposiciones y significados aprendidos de origen social, los cuales dan el marco de posibilidad a unas determinadas decisiones y prácticas económicas de los comportamientos razonables (que le lleva al autor asumir dicho concepto), en el sentido de que todas las personas estan dotadas de razón y siguen una lógica y un dinamismo totalmente compresibles si tenemos en cuenta su costrucción social». Esta lógica hace del comportamiento de los agentes un proceso de racionalización de una estrategia racionalizada en el comportamiento económico. (Paredes, M., 1994: 29) [11]

Los ejecutivos o directivos que soslayan o no identifican el conocimiento de la demanda en su dimensión de construcción social de sus consumidores, postergan o desaparecen del mercado. Lo cierto es que, además, aquellas empresas que fomentan la creatividad e innovación como valor son las que logran ejercer su liderazgo, irrumpiendo con nuevos productos y servicios, y permanecen en el mercado por más tiempo, satisfaciendo la demanda a partir del conocimiento que le proporciona la psicología económica, el que supone conjugar 
y conceder importancia a elementos que ya de por sí poseen suficiente entidad en el ámbito económico-empresarial: la formación con una potencialidad a desarrollar tanto en la configuración del capital humano como en la dinámica organizacional, visualizando y asumiendo la responsabilidad social; sin olvidar que el conocimiento de la naturaleza de la construcción social del consumidor y del capital intelectual del trabajador son componentes de los activos intangibles de la empresa.

Considerando que la visión que se tiene del usuario y sus necesidades, y su relación con la oferta informativa, así como de su comportamiento en la realidad y las tendencias que se identifican, que están bajo la influencia del entorno, asevera Rojas, J. (2004) [12] que la definición y dirección de los sistemas y entidades de información deben partir, irremisiblemente del usuario y sus necesidades. En estos tiempos, la evolución de los sistemas de información no está en dependencia del usuario, sino más bien de la tecnología.

La demanda de productos a escala global está cambiando, ubicándose habitualmente la satisfacción del usuario o cliente unida a la calidad y evaluación que efectúa como consumidor, partiendo de sus percepciones para la toma de decisiones que da inicio a su conducta. Tal es así, por ejemplo que «es probable que en el año 2020, cada persona del mundo desarrollado demande cerca de 29 kilogramos de carne y 63 litros de leche al año por encima de los 21 $\mathrm{kg}$ y 41 litros que demandaban en 1993, con proyecciones de que la demanda de cereales aumentará de $35 \%$ entre 1997 y $2020 \ldots$... y con otros aspectos de esperarse por cambios cualitativos en la demanda, por ejemplo la agricultura orgánica, aún cuando está lejos de ser sustituta de la agricultura convencional, será otro requerimiento, como lo es hoy la demanda de alimentos reforzados con hierro, yodo, vitamina A y micronutrientes, tales como el zinc, 1 riboflavina y el calcio para mitigar el impacto que tiene la deficiencia de estos elementos en la salud de los pueblos (Calis, R. 2004) [13].

Asimismo, la estructura de la demanda también se modificará, pues se espera que los países en desarrollo demandarán el doble de cereales y carne que los países desarrollados; y cobrará más fuerza la demanda por alimentos producidos con poco impacto ambiental y más saludables.

El fomento de interacciones y servicios diferenciados según ASOCAM (2002) [14] permite el desarrollo de alianzas estratégicas entre productores o diferentes actores, en tanto promueven la especialización, complementariedad y amplíen la base técnica y metodológica, facilitando a ello el acceso de los pequeños productores al mercado, favoreciendo su empoderamiento y capitalización campesina que será posible al involucrar nuevos actores como las universidades y los gobiernos locales. De igual manera la descentralización y el mejoramiento de la información relativa a consumos y a los mercados internos y externos (encuestas permanentes), como reorganizar la distribución interna de la producción (adoptar la tipificación uniforme de la producción), aumentar y diversificar los mercados externos: Producir o disponer productos de calidad; conocer los mercados externos, fomentar la promoción de las exportaciones.

El presente trabajo estudia la dimensión psicosocial de la percepción en el marco de la interacción interdependendiente y el empleo del conocimiento de demanda de la gestión comercial, que vincule la promoción de los derechos de la Comunidad Campesina Andina y de sus miembros, quienes establecerían una estrategia que otorge la diferencia y fortaleza 
basada en su cultura y conocimientos, e implementar aquéllos que desconozcan o le sean ajenos del comportamiento de la demanda. y salvaguardar la realidad de que «el comportamiento tradicional del productor sufre modificaciones de sus expectativas y de su cultura y costumbres tradicionales al modificarse la demanda de productos por un mercado creciente signado por la globalización» (Rebosio, G. y Rodríguez, E., 2001; Trivelli,C.; Von Hesse, M.; Diez, A. \& Del Castillo, L. 2001) [15], [16].

La percepción es un proceso cognitivo por el cual se optiene, captura, información proveniente del entorno, la que es utilizada para establecer planes de acción y tomar decisiones. En este proceso se establece a un segundo nivel, luego del sensitivo, el reconocimiento que permite la expresión de significaciones al establecer categorías.

\section{Variables de estudio}

- Percepción al conocimiento de la demanda de productos.

- Percepción hacia la interdependencia económica.

- Miembros del caserío Queral de la Comunidad campesina Santa Cruz, mayores de 14 años: Edad, género y estudios.

- Unidad de observación: Un representante de cada familia.

\section{Hipótesis}

$\mathrm{H}$ : Existe relación entre el conocimiento de la demanda y percepciones hacia la interdependencia económica en el caserío Queral en Caraz, Ancash.

\section{MÉTODO}

Diseño: Es una investigación descriptiva de tipo correlacional. Aplicativa.

\section{Territorio y geografía}

El territorio del caserío Queral, donde se ha realizado la investigación, depende políticamente de la Comunidad campesina Cruz de Mayo; ubicada administrativamente en el distrito de Caraz, provincia de Huaylas; el que comparte al igual que los otros tres caseríos el puesto de salud, ubicado en el caserío de Pampacocha. En cada investigación se firmó un acuerdo de colaboración entre los representantes del caserío y los investigadores.

Caraz es la población de mayor influencia de la zona y capital de la provincia. Desde Caraz, la zona ocupada por los caseríos, se localiza a unos $8 \mathrm{~km}$ lineales con orientación 36 Ú Norte. Las coordenadas de localización de la zona se sitúan aproximadamente en los 77Ú 47' longitud Oeste y 9Ú 00' de latitud Sur.

Este caserío se ubica en la falda de los nevados más elevados de la Cordillera Blanca, en la zona occidental de los Andes peruanos: Huandoy $(6395 \mathrm{msnm})$ y ligeramente al sureste Huascarán (6768 msnm). En paralelo discurre la Cordillera Negra, que supera los 5000 msnm. Entre ambas cordilleras se encuentra el Callejón de Huaylas, un fértil valle agrícola bañado por el río Santa. 
Los caseríos de la Comunidad Cruz de Mayo carecen de infraestructura básica: existe un grifo de agua por familia, al momento están instalando la corriente eléctrica, las fuentes energéticas son las tradicionales, fundamentalmente el fuego de madera y velas. La infraestructura vial se reduce a un camino carrozable afirmado, que llega hasta Pampacocha (H»3300 msnm) pasando por Miramar (H»3150 msnm) y Queral $(\mathrm{H} » 3200 \mathrm{msnm})$. A Chinchay (H»3300 msnm) no llega, se accede solo por camino de herradura desde Pampacocha durante 10 minutos. No hay línea regular de viajeros, la movilidad es privada. El transporte colectivo se activa a primera hora de la mañana cuando hay personas suficientes para rentabilizar la subida de un auto de 5 plazas, en el que suben 8 personas (en ocasiones hasta 12), o una «combi». Siempre se puede alquilar un taxi a nivel privado, pero esta opción escapa económicamente de los miembros de la comunidad. En el invierno, la duración del trayecto es de algo más de una hora, en tiempo de lluvias se incrementa considerablemente.

También se dispone de un camino de herradura (no apto para ningún tipo de vehículo) que supera los $1200 \mathrm{~m}$ de desnivel entre Caraz y Pampacocha en apenas $8 \mathrm{~km}$. Este desnivel, junto a los cortados y su estrechez lo hacen un camino muy difícil para quien tenga falta de costumbre y no lo conozca. Un miembro de la comunidad joven y sano recorre el trayecto bajando en aproximadamente 45 minutos a una hora, tardando más de dos horas y media los maestros o un excursionista. La subida, confiesan los comuneros, se recorre en dos horas; los maestros apuntan que no suben andando, de hacerlo les llevaría cerca de las cinco horas.

Existe una escuela de primaria por caserío. La educación secundaria recién en el presente año se realiza en Pampacocha, sin la presencia del Proyecto Huascarán, y los alumnos acceden a los centros a pie y en las condiciones antes descritas.

Entre los productos agrícolas que les solventa «en algo» y para adquirir bienes de la zona de influencia es la arveja criolla, aproximadamente 8 sacos semanales en época de producción.

La ubicación de la zona de intervención se concreta con los siguientes datos: Perú, hemisferio sur del Continente americano. Topográficamente en la región de sierra. Políticamente en la Región Ancash, departamento de Ancash, provincia de Huaylas, distrito de Caraz. Los caseríos son asentamientos humanos estables vinculados legalmente a una comunidad campesina.

Las comunidades campesinas son organizaciones de interés público, con existencia legal y personería jurídica, integradas por familias que habitan y controlan determinados territorios ligadas por vínculos ancestrales, sociales, económicos y culturales, expresados en la propiedad comunal de la tierra, el trabajo comunal, la ayuda mutua, el gobierno democrático y el desarrollo de actividades multisectoriales, cuyos fines se orientan a la realización plena de sus miembros (Ley 24656, Art. 2). Se puede apreciar en el Anexo N. ${ }^{\circ} 1$.

Población: Por tratarse de un caserío con pocos habitantes se ha decidido que sean entrevistados en su totalidad, tomando como unidad de observación a la familia, representada por un sujeto en calidad de jefe de familia o representante de ella.

En total son 51 familias, que constituyen en total 125 miembros, de 14 hasta los 59 años, edad promedio de 33 para las mujeres y 35 para los varones. La PEA estudiada son 51 miembros pertenecientes a cada familia que tienen la potestad para decidir; de los cuales 20 son mujeres y 31 varones, de estos a su vez 17 mujeres y 26 varones son casados. La 
condición de membresía es 24 comuneros, 8 hijos de comuneros y 19 propietarios; con el nivel educativo de 26 con primaria completa, 17 con primaria incompleta y 7 iletrados.

\section{Instrumento}

La recolección de datos mediante el uso de cuestionarios preparados para esta investigación. Un cuestionario sobre la Percepción del conocimiento de la demanda de productos y su aceptación en diversos mercados y una Escala de Percepciones a la Interdeoendencia económica.

Previamente, para el diseño de los instrumentos definitivos, se realizó una entrevista semiestructurada; a modo de ejemplo, indicamos las siguientes preguntas que forman parte de ella:

1. (4) ¿Cuáles son los motivos que nos dificultaron o impidieron, en ocasiones anteriores, hacer posible que nos uniéramos entre familias para vender?

2. (6) ¿Cuáles son las causas que nos resta a que desconfiemos entre nosostros mismos como familias para vender en conjunto?

3. (8) ¿Es más fuerte el individualismo que tenemos para los negocios?

4. (9) ¿Queremos diferenciarnos o tenemos vergüenza para obtener ganancias económicas?

a) Escala de Percepción hacia la Interdependencia Económica: Mide tres factores independientemente, cuya validez se indica con sus respectivos ítemes: 1) Actitud hacia la interdependencia por Infraestructura de soporte para la producción: 1, 3, 7, 10 $(0,57) ; 2)$ Actitud hacia la interdependencia, gestión de la producción: $2,9,12,13$ $(0,50)$; y 3) Actitud hacia la interdependencia por mercado: Percepción de la oferta: 8 , $11(0,59)$. La escala tiene una confiabilidad de Alfa de Cronbach Total $=0,78$ (ver Tabla 1).

b) Cuestionario de percepción del donocimiento de la demanda: Su confiabilidad Alfa de Cronbach 0,93., con las siguientes áreas y sus respectivos ítemes: 1) Conocimiento de los gustos del consumidor: $1,12,(0,86)$; 2) Conocimiento de los precios: 2, 5, 13 $(0,78)$; 3) Conocimiento de la competencia: 3,$15 ; 4)$ Conocimiento de su posicionamiento: $4,9,11,16,(0,78)$; 5) Oferta: $6,8,18,19(0,72)$; y 6) Canal de comercialización: $7,13,(0,72)$ (ver Cuadro 1$)$.

\section{Procedimiento}

1. Se realizaron once entrevistas semiestructuradas con el objetivo de discriminar los indicadores básicos para la escala y el cuestionario de percepción del conocimiento de la demanda e interdependencia económica respectivamente. Entrevista efectuada con aquellos miembros que tienen mayor dominio del castellano, asimismo se buscó focalizar lo observado en las relaciones de reciprocidad que se da entre los miembros de la comunidad en los diferentes aspectos de convivencia en la comunidad y no eran al menos evidentes para los negocios o actividades económicas con retribución pecuniaria o monetaria. 2) Previas coordinaciones y aprobación de los integrantes participantes en el presente estudio, se aplicaron en quechua las dos escalas, impresas en una sola hoja a fin de disponer del dato de la percepción y actitud medida por cada escala a cada unidad de observación por un miembro del caserío del lugar. 


\section{RESULTADOS}

Los resultados que a continuación se presentan tanto en las Tablas 1 y 2 contienen la confiabilidad de los instrumentos de medición: Escala de Percepción hacia la Interdependencia económica y el Cuestionario de percepción del conocimiento de la demanda.

Tabla 1. Confiabilidad de la percepción hacia la interdependencia económica miembros de Queral.

\begin{tabular}{|c|c|c|c|c|}
\hline Área & M & D. E. & $\begin{array}{c}\text { Correlación item-test } \\
\text { corregido }\end{array}$ & $\begin{array}{l}\text { Alfa de } \\
\text { Cronbach }\end{array}$ \\
\hline \multicolumn{5}{|c|}{ Infraestructura } \\
\hline inter10 & 3,62 & ,490 & ,424 & \\
\hline inter3 & 3,36 & ,485 & ,247 & \\
\hline inter1 & 3,72 & ,454 & ,175 & \\
\hline inter7 & 3,66 & ,479 & ,346 & 0,57 \\
\hline \multicolumn{5}{|c|}{ Gestión de la producción } \\
\hline inter2 & 3,46 &, 503 & ,212 & \\
\hline inter9 & 3,20 & ,404 & ,305 & \\
\hline inter12 & 3,52 &, 505 & ,315 & \\
\hline inter13 & 3,40 & ,495 &, 368 & 0,50 \\
\hline \multicolumn{5}{|c|}{ Mercado - Percepción de la oferta } \\
\hline inter8 & 3,18 & ,388 & ,414 & \\
\hline inter11 & 3,20 & ,404 & ,357 & 0,59 \\
\hline \multicolumn{5}{|c|}{ Alfa de Cronbach Total $=0,78$} \\
\hline
\end{tabular}

Los resultados descritos a continuación en la Tabla 2 de la percepción conocimiento arrojan:

El nivel total logrado en el factor cognitivo y categorización que proporciona la percepción del Conocimiento de la demanda total $(8,11)$ es bajo porque el total de preguntas es 19 , y el puntaje obtenido más bajo se da en la percepción del conocimiento de la demanda en el área de Precios al consumidor $(1,06)$. La población estudiada estima en darle un mayor valor, en el siguiente orden a: $1^{\circ}$ Conocimiento de la demanda por la oferta; $2^{\circ}$ Conocimiento de su posicionamiento; $3^{\circ}$ Conocimiento de la demanda según el canal de comercialización; $4^{\circ}$ Conocimiento de la competencia; $5^{\circ}$ Conocimiento de la demanda por los gustos del consumidor; $6^{\circ}$ Conocimiento de la demanda por los precios. 
Tabla 2. Confiabilidad del conocimiento de la demanda de productos de Queral.

\begin{tabular}{|c|c|c|c|c|}
\hline $\begin{array}{c}\text { Área de } \\
\text { conocimientos }\end{array}$ & M & D. E. & $\begin{array}{c}\text { Correlación ítem-test } \\
\text { corregido }\end{array}$ & $\begin{array}{c}\text { Alfa de } \\
\text { Cronbach }\end{array}$ \\
\hline \multicolumn{5}{|c|}{ Gustos del consumidor } \\
\hline demanda1 & 1,12 & ,328 & ,324 & \\
\hline demanda4 & 1,16 & ,370 &, 595 & 0,86 \\
\hline \multicolumn{5}{|c|}{ Precios al consumidor } \\
\hline demanda2 & 1,06 & ,240 &, 584 & \\
\hline demanda5 & 1,06 & ,240 &, 584 & 0,78 \\
\hline \multicolumn{5}{|l|}{ Competencia } \\
\hline demanda3 & 1,06 & ,240 & ,461 & \\
\hline demanda15 & 1,22 & ,616 & ,461 & 0,64 \\
\hline \multicolumn{5}{|c|}{ Posicionamiento productos } \\
\hline demanda9 & 2,08 & ,274 & ,821 & \\
\hline demanda11 & 1,82 &, 523 & ,355 & \\
\hline demanda16 & 1,40 & 670 & ,619 & \\
\hline demanda17 & 1,14 &, 572 & ,806 & 0,78 \\
\hline \multicolumn{5}{|l|}{ Canal de ventas } \\
\hline demanda7 & 2,06 & ,242 & ,397 & \\
\hline demanda13 & 1,04 & ,286 & ,286 & 0,72 \\
\hline \multicolumn{5}{|l|}{ Oferta } \\
\hline demanda6 & 2,06 &, 240 & 675 & \\
\hline demanda8 & 2,10 & ,364 & 622 & \\
\hline demanda18 & 1,14 &, 572 & 875 & \\
\hline demanda19 & 1,14 &, 572 & ,875 & 0,74 \\
\hline \multicolumn{5}{|c|}{ Alfa de Cronbach total $=0,90$} \\
\hline
\end{tabular}

El resultado observado referente a la Percepción hacia la interdependencia de los miembros del caserío de Queral es alto, se ubica entre los valores 3,19 a 3,59 en una escala de 4 valores.

Así los miembros clasifican y priorizan la actitud a la interdependencia económica en el siguiente orden: $1^{\circ}$ Infraestructura $(3,59)$; Gestión a la producción $(3,39)$; y Percepción a la oferta $(3,19)$. 
Tabla 3. Análisis de la bondad de ajuste a la curva normal de la percepción hacia la interdependencia económica y conocimiento de la demanda de productos.

\begin{tabular}{lccc}
\hline \multicolumn{1}{c}{ Variables (áreas) } & M & D. E. & Z K-S \\
\hline \multicolumn{1}{l}{ Percepción del conocimiento de la demanda } & & \\
\hline Gusto & 1,14 & 0,30372 & 3,377 \\
Precios & 1,06 & 0,21759 & 3,738 \\
Competencia & 1,14 & 0,3785 & 3,424 \\
Posicionamiento & 1,61 & 0,41082 & 2,443 \\
Canal & 1,55 & 0,23146 & 3,716 \\
Oferta & 1,61 & 0,38518 & 3,623 \\
Demanda total & 8,11 & 1,66381 & 2,591 \\
\hline Percepción hacia la interdependencia & & & \\
\hline Infraestructura & 3,59 & 0,31445 & 1,517 \\
Gestión de la producción & 3,395 & 0,30342 & 1,723 \\
\hline Percepción de la oferta & 3,19 & 0,33335 & 3,081 \\
\hline$N=51$ & & &
\end{tabular}

Entre los resultados de la correlación significativa entre la Percepción del Conocimiento de la demanda con la Percepción hacia la interdependencia económica, se observan en la Tabla 4:

1. Conocimiento de la demanda por los gustos del consumidor con percepción hacia la interdependencia por gestión a la producción $(-0,27)$.

2. Conocimiento de la demanda por los precios con percepción hacia la interdependencia por la infraestructura para la producción $(-0,27)$.

3. Conocimiento de la demanda según el canal de comercialización con percepción hacia la interdependencia por la infraestructura para la producción $(-0,34)$.

4. Conocimiento de la demanda según el canal de comercialización con percepción hacia la interdependencia por la gestión de la producción $(-0,25)$.

5. Conocimiento de la demanda por la oferta con percepción hacia la interdependencia por la percepción de la oferta $(0,29)$. 
Tabla 4. Matriz de correlaciones del coeficiente Tau de Kendall entre la percepción hacia la interdependencia económica y el conocimiento de la demanda de productos.

\begin{tabular}{lcccc}
\hline $\begin{array}{l}\text { Conocimiento } \\
\text { de la demanda }\end{array}$ & Infraestructura & $\begin{array}{c}\text { Procesos del } \\
\text { producto }\end{array}$ & $\begin{array}{c}\text { Percepción } \\
\text { de la oferta }\end{array}$ & $\begin{array}{c}\text { Total } \\
\text { interdependencia }\end{array}$ \\
\hline Gustos & $-0,17$ & $-0,27 *$ & 0,15 & $-0,16$ \\
Precios & $-0,27 *$ & $-0,15$ & $-0,04$ & $-0,21$ \\
Competencia & $-0,14$ & $-0,18$ & 0,09 & $-0,10$ \\
Posicionamiento & $-0,06$ & 0,02 & $-0,08$ & $-0,08$ \\
Canal & $-0,34 * *$ & $-0,25 *$ & 0,06 & $-0,18$ \\
Oferta & $-0,16$ & $-0,03$ & $0,29 *$ & 0,05 \\
$\begin{array}{l}\text { Demanda Total } \\
\text { N }\end{array} \quad-0,14$ & $-0,18$ & 0,20 & $-0,05$ \\
** significancia $<0,01$ & & &
\end{tabular}

\section{DISCUSIÓN}

Los resultados, por tanto, nos indican que en el caserío Queral pueden lograr cadenas productivas (alianzas o interdependencias empresariales) entre sus miembros por su percepción del conocimiento de la demanda de la oferta y la percepción hacia la interdependencia por la percepción de la oferta $(0,29)$.

Esta variable identificada se considera básica para desarrollo económico del Caserío, sustentada por los fuertes lazos culturales y sociales manifestados en su identidad y solidaridad, dimensiones prioritarias en la representación de su construcción social de la organización de la comunidad campesina andina del caserío de Queral y que no se observan en la actividad económica con lucro explícito.

Así, todos los procesos productivos dirigidos a un beneficio económico serán orientados hacia el logro de la oferta que demanda el consumidor, otorgando probabilidades mayores de generar relaciones interdependientes económicas.

Esta relación positiva, única, entre la Percepción del conocimiento de la demanda, referente al conocimiento de la oferta de sus productos, con la Percepción hacia la interdependencia por la Percepción de la oferta nos indica que, para lograr una mejor sostenibilidad en la gestión de la actividad económica del caserío, tendrá que estar sustentada de manera sostenida mediante las tareas que realicen los miembros dirigidas a la obtención de la calidad y volumen de la oferta de sus productos para el consumidor. Teniendo la partida por tareas referidas a la infraestructura.

Se fundamenta en que la percepción es el proceso psicológico mediante el cual el sujeto otorga significado a todo aquello que le rodea y de lo que es consciente y, a partir de ella, se forma la actitud que se expresa en una tendencia factible a modificar el comportamiento económico del caserío, sin descuidar y en tiempo real, para ello, se hace indispensable el diseño y desarrollo de programas de capacitación orientados a la información sobre el contexto económico en el que van a insertarse y el adiestramiento de sus actores en procesos 
productivos que perciban la obtención de una oferta según las normas y especificaciones del mercado; procesos de gestión de recursos humanos entendidos como inversión con altos niveles de probabilidades de recuperación.

Es de precisar que se observa una relación significativa inversa en las demás áreas, parafraseando: a menor conocimiento de la demanda mayor percepción hacia la interdependencia económica para el caserío. Nivel cognitivo que expresaron como «desconfianza en los negocios» «viveza y engaño -usar el dinero aún cuando lo repongan después-». Especialmente se observa la relación inversa entre la percepción hacia la interdependencia económica por el área de procesos de productos con las áreas de la percepción del conocimiento de la demanda; en tanto que las faenas y trabajos comunales son una actividad social y cultural por primacía, no tiene al parecer una categorización de percepción económica de usufructo.

\section{CONCLUSIONES}

1. Al contrastar la $\mathrm{H}$, no se encuentra una relación significativa entre la Percepción del conocimiento de la demanda y percepción hacia la interdependencia económica en el caserio Queral en Caraz, Ancash.

2. La percepción del conocimiento de la demanda es bajo y la percepción hacia la intedependencia económica es alto.

3. En la interdependencia económica entre los miembros de Queral categorizan a la infraestructura con primer lugar.

4. Hay una relación significativa positiva entre la percepción del conocimiento de la demanda del área percepción de la oferta con la interdependencia económica hacia la percepción de la oferta; indicador más significativo para lograr alianzas económicas para digir una gestión competitiva a un mediano plazo.

Asi mismo, se lograron los siguientes objetivos:

1. Ampliar el conocimiento académico de la psicología social organizacional y psicología económica de las organizaciones de las comunidades andinas.

2. Identificar las percepciones, mediadoras de la interacción interdependiente de los miembros del caserío Queral para la formación de relaciones interdependientes económicas (cadenas productivas).

3. Determinar el nivel percepción del conocimiento de la demanda de sus productos significativos del caserío Queral.

4. Disponer de dos instrumentos con nivel de confiabilidad de 0,78 y 0,90 , respectivamente.

\section{REFERENCIAS BIBLIOGRÁFICAS}

1. Paredes, M. y De Miguel, J. Ma ${ }^{\mathrm{a}}$. (2004). Construcción social de la comunidad para el desarrollo del Callejón de Huaylas, Caraz. Revista de Investigación en Psicología, Vol. 7(1), 81-102, Instituto de Investigaciones Psicológicas, Facultad de Psicología, Universidad Nacional Mayor de San Marcos, Lima. 
2. Paredes, M.; De Miguel, J. Ma.; Vicuña, L. y Hernández, H. (2005). «El papel de la construcción social de la organización con respecto a la percepción de las condiciones de trabajo y el manejo del medio ambiente en 4 caseríos de una comunidad campesina andina-Caraz, Ancash. Revista del Instituto de Investigación de la Facultad de Ingeniería Geológica, Minería, Metalúrgica y Geográfica Vol. 8 N¹6, Universidad Nacional Mayor de San Marcos, Lima.

3. McClelland, D. y Winter, D. (1970). Cómo se motiva el éxito económico, $1 .^{\mathrm{a}}$ edic. en español. México D.F.: Uteha.

4. Paredes, M. (2001). La interdependencia empresarial y la calidad de gestión. Revista de Investigaciones en Psicología $N^{0}$ 2. Instituto de Investigaciones Psicológicas, Facultad de Psicología, Universidad Nacional Mayor de San Marcos, Lima.

5. Holahan, Ch. (1996). Psicología ambiental. Un enfoque general. México: Editorial Limusa S.A.

6. Katona, G, (1965). Análisis psicológico del comportamiento económico. Madrid: Rialp S.A. Español.

7. Paredes, M. (1977). La interdependencia empresarial, factor de calidad en la GestiónCaso Sector Empresarial Pesquero Peruano. Tesis para optar el Grado de Magíster en Administración Mención Comercio Internacional. UNMSM / Unidad de Post Grado Facultad de Ciencias Administrativas.

8. Paredes Tarazona, Mildred (2002). Intereses, motivaciones y percepciones de empresarios pesqueros peruanos hacia la pesca sostenible. Revista del Instituto de Investigación vol $5 \mathrm{~N}{ }^{\circ} 9$, Facultad de Ingeniería Geológica, Minería, Metalúrgica y Geográfica, Universidad Nacional Mayor de San Marcos, Lima.

9. De Miguel, J. Ma. (2004). Dimensiones psicosociales del desarrollo local. Psicología y derechos humanos. En: De la Corte, Luis; Blanco, Amalio; Sabucedo, José Manuel. Paz y conflictos. Barcelona: Editorial Fundación Cultura de Paz \& Icaria. Antrazyt.

10. Juanola i Hospital, Eduard. (2004). Introducción a la psicología económica. Gil Juárez, Adriana (Coord.), Psicología económica y del comportamiento del consumidor. Barcelona: Editorial Udc.

11. Paredes Tarazona, Mildred (1994). El proceso del comportamiento de las unidades económicas. Documento de Trabajo. UNMSM, Facultad de Ciencias Administrativas/ Unidad de Post Grado, Curso de Finanzas Internacionales. (Biblioteca de la Facultad de Psicología).

12. Rojas, J. (2004). «Evolución de las necesidades de información: oferta y demanda de productos». Universidad de La Habana, Facultad de Comunicación. www.congresoinfo.cu/User/File/Info/Info2004/Ponencias.

13. Calis, R. (2004). Agricultura y desarrollo: tendencias del futuro. En: Revista Actualidad económica Año XVIII, 307 del 5 al 28 de noviembre 2004. www.actualidad.co.cr/307/ 83.rafael_celis.html.

14. ASOCAM. (2002). Aspectos claves para el acceso a mercado por parte de pequeños productores. Serie: Reflexiones y aprendizajes. Abril. 2002. www.oas.org/osde/ publications/Unit/oea21s/ch23.htm. 
PeRCEPCIÓN del CONOCIMIENTO de LA deMANDA DE PRODUCTOS Y PERCEPCIÓN HACIA LA INTERDEPENDENCIA ECONÓMICA EN EL CASERÍO QueRAL

15. Rebosio, G. y Rodríguez, E. (2001). Ingreso campesino y compras estatales de alimentos en el Perú. Lima: CIES-CEDEP.

16. Trivelli, C.; VonHesse, M.; Diez, A. y Del Castillo, L. (2000). Desafíos del desarrollo rural en el Perú. Lima: CIES. 